NASA Technical Memorandum 102293

ICOMP-89-18

\title{
Calculation of Reattaching Shear Layers in Divergent Channel With a Multiple- Time-Scale Turbulence Model
}

S.-W. Kim

Institute for Computational Mechanics in Propulsion

Lewis Research Center

Cleveland, Ohio

August 1989

\section{Mnsh}

(NASA-TM-102293) CALCULATION OF REATTACHING

SHEAR LAYERS IN DIVERGENT CHANNEL WITH A

MULTIPLE-TIME-SCALE TURBULENCE MUDEL (NASA.

Lewis Research (enter) $27 \mathrm{D}$ CSCL 200

$\begin{array}{ll}\text { Unclas } \\ 03 / 34 & 0225964\end{array}$


CALCULATION OF REATTACHING SHEAR LAYERS IN DIVERGENT CHANNEL WITH

A MULTIPLE-TIME-SCALE TURBULENCE MODEL

\author{
S. -W. Kim* \\ Institute for Computational Mechanics in Propulsion \\ Lewis Research Center \\ Cleveland, Ohio 44135
}

\begin{abstract}
SUMMARY
Numerical calculations of turbulent reattaching shear layers in a divergent channel are presented. The turbulence is described by a multiple-time-scale turbulence model. The turbulent flow equations are solved by a control-volume based finite difference method. The computational results are compared with those obtained using $k-\varepsilon$ zurbulence models and algebraic Reynolds stress turbulence models. It is shown that the multiple-time-scale turbulence model yields significantly improved computational results than the other turbulence models in the region where the turbulence is in a strongly inequilibrium state.
\end{abstract}

*Work funded under Space Act Agreement C99066G. 


\section{NOMENCLATURE}

$A_{u}$

$\mathrm{A}_{\mathrm{v}}$

$c_{f}$

$c_{p}$

$c_{\mu \mathrm{f}}$

$c_{p \ell}$

$c_{t \ell}$

$c_{\mu \mathrm{f}}$

$\mathrm{f}_{\mu}$

$f_{\epsilon}$

$\mathrm{H}$

$\mathrm{k}$

$k_{p}$

$k_{t}$

$\mathrm{p}$

$P_{r}$

$\mathrm{R}_{\mathrm{t}}$

$\mathrm{U}_{\infty}$

$u_{j}$

$u_{r}$

$\overline{u_{i}{ }^{\prime} u_{j}{ }^{\prime}}$

v

$x_{j}$

$\mathrm{x}_{\mathrm{R}}$

$\mathrm{y}^{+}$

$\alpha$ coefficient for tangential velocity correction

coefficient for transverse velocity correction

friction coefficient $\left(=\tau_{w} /\left(0.5 \rho \mathrm{U}_{\infty}^{2}\right)\right)$

pressure coefficient $\left(=\mathrm{p} /\left(0.5 \rho \mathrm{U}_{\infty}{ }^{2}\right)\right)$

constant coefficient for eddy viscosity equation $(=0.09)$

turbulence model constants for $\epsilon_{\mathrm{p}}$ equation $(\ell=1,3)$

turbulence model constants for $\epsilon_{t}$ equation $(l=1,3)$

constant coefficient $(=0.09)$

wall damping function for eddy viscosity equation

wall damping function for $\epsilon_{\mathrm{w}}$ equation

height of backward-facing step

turbulent kinetic energy $\left(k=k_{p}+k_{t}\right)$

turbulent kinetic energy of eddies in production range

turbulent kinetic energy of eddies in dissipation range

pressure

production rate of turbulent kinetic energy

turbulent Reynolds number $\left(-k^{2} /\left(\nu \epsilon_{1}\right)\right)$

inlet flow velocity

time averaged velocity $(=\{u, v\})$

friction velocity $\left(=\sqrt{ }\left(r_{\mathrm{w}} / \rho\right)\right)$

Reynolds stress $(i=1,2,3$ and $j=1,2,3)$

velocity vector $(-\{u, v\})$

spatial coordinates $(=\{x, y, z\})$

reattachment location

wall coordinate $\left(=u_{\tau} \mathrm{y} / \nu\right)$

deflection angle of the top wall 


$\begin{array}{ll}\epsilon_{\mathrm{p}} & \text { energy transfer rate from production range to } \\ \epsilon_{\mathrm{t}} & \text { dissipation range } \\ \epsilon_{1} & \text { dissipation rate of turbulent kinetic energy } \\ \kappa & \text { von Karman constant }(-0.41) \\ \mu & \text { molecular viscosity } \\ \mu_{\mathrm{e}} & \text { effective viscosity }\left(=\mu+\mu_{t}\right) \\ \mu_{\mathrm{t}} & \text { turbulent viscosity } \\ \nu & \text { kinematic viscosity of fluid } \\ \nu_{t} & \text { turbulent eddy viscosity } \\ \rho & \text { density } \\ \sigma_{\mathrm{kp}} & \text { turbulent Prandtl number for } k_{\mathrm{p}} \text { equation } \\ \sigma_{\mathrm{kt}} & \text { turbulent Prandtl number for } k_{t} \text { equations } \\ \sigma_{\epsilon \mathrm{p}} & \text { turbulent Prandtl number for } \epsilon_{\mathrm{p}} \text { equation } \\ \sigma_{\epsilon t} & \text { turbulent Prandtl number for } \epsilon_{\mathrm{t}} \text { equation } \\ \tau_{\mathrm{w}} & \text { wall shearing stress }\end{array}$




\section{INTRODUCTION}

The experimental study of a reattaching shear layer in a divergent channel [1] was designed to test the predictive capability of various turbulence models, to identify any deficiency in turbulence closure models, and thus to improve predictive capability of turbulence models. The flow geometry is shown in Figure 1. The height of the backward-facing step is smaller than the boundary layer thickness of the incoming flow. Abrupt breakdown of the boundary layer generated a strongly inequilibrium turbulent flow. Furthermore, a strong pressure gradient was generated by varying the divergence angle of the top wall to study its effect on the development of the turbulence field, especially the Reynolds stress, and the reattachment process. A number of turbulence models, such as $\mathrm{k}-\epsilon$ turbulence models and algebraic Reynolds stress turbulence models (ARSM), were shown to yield poor computational results for the flow $[1,2]$. It is also shown in References 1 and 2 that a modified ARSM, with modifications in the dissipation rate equation, yielded computational results which are in good agreement with measured data. However, generality of the improved predictive capability for other complex turbulent flows has not been shown yet.

It has been shown previously that the high Reynolds number multiple-time-scale turbulence model yields accurate computational results for a number of complex turbulent flows such as a wall jet flow, a wake-boundary layer interaction flow, a confined coaxial jet without swirl and a confined coaxial swirling jet to name a few $[3,4]$. In the single-time-scale turbulence models such as $k-\epsilon$ turbulence models, algebraic stress turbulence models, and Reynolds stress turbulence models, a single time scale is used to express both the turbulent transport and the 
dissipation of the turbulent kinetic energy. However, this practice is inconsistent with physically observed turbulence in the sense that the turbulent transport is related to the time scale of energy containing large eddies and the dissipation of turbulent kinetic energy is related to the time scale of fine scale eddies in the dissipation range. The single-time-scale turbulence models yield reasonably accurate computational results for simple turbulent flows; however, the predictive capability degenerates rapidly as turbulent flows to be solved become more complex. In the multiple-time-scale turbulence models [3-7], the turbulent transport of mass and momentum is described using the time scale of the large eddies and the dissipation rate is described using the time scale of the fine-scale eddies. The improved computational results obtained using the multiple-time-scale turbulence model for complex turbulent flows can be attributed to the physically consistent nature of the turbulence models discussed above.

In numerical calculations of turbulent flows, wall function methods are most frequently used to model the near-wall region. These methods have been derived from the logarithmic velocity profile based on experimental observations that the turbulence in the near-wall region can be described in terms of the wall shearing stress. Therefore, these methods are not valid if the logarithmic velocity profile no longer prevails in the near-wall region. For example, the logarithmic velocity profile no longer prevails in the near-wall or in the wake reglons of unsteady turbulent flows [8], therefore wall function methods can not be applied. Many other cases for which the wall function methods are invalid can be found in References 9 and 10. Due to this limited applicability of the wall function methods, numerous alternative approaches have been proposed. In the 
alternative approaches, the near-wall low turbulent Reynolds number region is included into numerical analyses to overcome the shortcomings of the wall functions methods. Various turbulence models which include the near-wall low turbulence region can be classified as two-layer (or multi-layer) turbulence models [11] and low Reynolds number turbulence models [10] based on the way the near-wall region is treated. More detailed discussion on the advantages and disadvantages of various near-wall turbulence models can be found in References 9 and 10 .

In the present study, the near-wall turbulence is described by a "partially low Reynolds number approach." In the model [9], only the turbulent kinetic energy equations are extended to include the near-wall low turbulence region and the energy transfer rate and the dissipation rate inside the near-wa11 layer are obtained from algebraic equations. The algebraic equations were obtained from a k-equation turbulence model [12]. It would be appropriate to classify the method as a "partially low Reynolds number approach" to distinguish it from other classes of methods. This approach was first used in Chen and Patel to solve turbulent flows over airfoils [13]. Advantages of the partially low Reynolds number approach over the other methods can be summarized as follows. The turbulence length scale of the external flows is related to the flow field characteristics [14]. On the other hand, the turbulence length scale of boundary layer flows is strongly related to the normal distance from the wall. This characteristic of the wall bounded turbulent flows can be described quite naturally by the present class of turbulence models. The low Reynolds number turbulence models can also be used to describe the wall bounded turbulent flows; however, more grid points have to be used to resolve the steep dissipation rate in the near-wall region. It is also interesting to 
note that various similar k-equation turbulence models, which form the basis of the present near-wall turbulence model, yield accurate computational results for a class of simple turbulent boundary layer flows [15], turbulent flows with drag reduction [16], and fully developed unsteady turbulent pipe flows [8]. However, the k-equation turbulence model itself is less useful for separated and/or swirling turbulent flows with complex geometry due to lack of a systematic method to evaluate the turbulence length scale. Development of the near-wall turbulence model and its application to fully developed turbulent channel and pipe flows can be found in Reference 9. It has been shown in the reference that the present near-wall turbulence model can resolve the over-shoot phenomena of the turbulent kinetic energy and the dissipation rate in the region very close to the wall and that significantly improved computational results for the turbulence structure in the near-wall region are obtained. Incorporation of the same near-wall turbulence model into a $k-\epsilon$ turbulence model and its application to complex turbulent flows such as a supersonic turbulent flow over a compression ramp and a transonic flow over an axisymmetric curved hill can be found in References 17 and 18 , respectively.

The numerical method used herein is based on the pressure correction method [19] which has been used most extensively to solve incompressible flows the domain of which can be discretized by an orthogonal mesh. However, the present numerical method is applicable for both incompressible and compressible flows with arbitrary, complex geometries. The capability to solve compressible flows is achieved by including a convective incremental pressure term into the pressure correction equation $[17,18]$. In the method, the velocities are located at the same grid points and the pressure is located at the centroid of the cell formed by the four adjacent 
velocity grid points. This grid layout was found to be quite suitable to solve flows with complex geometries [17]. The accuracy and the convergence nature of the numerical method have been demonstrated by solving a number of flow cases. The example problems considered in References 17 and 18 include: a developing channel flow, a developing pipe flow, a two-dimensional laminar flow in a 90 degree bent channel, polar cavity flows, a turbulent supersonic flow over a compression ramp, and a shock wave - turbulent boundary layer interaction in transonic flow over a curved hill. It was found that the numerical method used herein yielded accurate computational results even when highly skewed, unequally spaced, curved grids were used.

\section{TURBULENT FLOW EQUATIONS}

The incompressible turbulent flow equations are given as;

$$
\frac{\partial}{\partial x}(\rho \mathrm{u})+\frac{\partial}{\partial y}(\rho \mathrm{v})=0
$$

$$
\frac{\partial}{\partial x}(\rho u v)+\frac{\partial}{\partial y}(\rho v u)=\frac{\partial}{\partial x}\left(2 \mu_{e} \frac{\partial u}{\partial x}\right)+\frac{\partial}{\partial y}\left(\mu_{e}\left(\frac{\partial u}{\partial y}+\frac{\partial v}{\partial x}\right)\right)-\frac{\partial p}{\partial x}
$$

$$
\frac{\partial}{\partial x}(\rho \mathrm{uv})+\frac{\partial}{\partial y}(\rho v v)-\frac{\partial}{\partial x}\left(\mu_{\mathrm{e}}\left(\frac{\partial u}{\partial y}+\frac{\partial v}{\partial x}\right)\right)+\frac{\partial}{\partial y}\left(2 \mu_{e} \frac{\partial v}{\partial y}\right)-\frac{\partial p}{\partial y}
$$

where eqs. (1-3) follow from the conservation of mass, u-momentum, and $v$-momentum, respectively. In numerical calculation, the conservation of mass equation is replaced by a pressure correction equation given as: 


$$
\frac{\partial}{\partial x}\left(\frac{\mathrm{u}^{*}}{\mathrm{RT}} \mathrm{p}^{\prime}\right)+\frac{\partial}{\partial \mathrm{y}}\left(\frac{\mathrm{v}^{*}}{\mathrm{RT}} \mathrm{p}^{\prime}\right)=\frac{\partial}{\partial \mathrm{x}}\left(\rho^{*} \mathrm{~A}_{\mathrm{u}} \frac{\partial \mathrm{p}^{\prime}}{\partial \mathrm{x}}\right)+\frac{\partial}{\partial \mathrm{y}}\left(\rho^{*} \mathrm{~A}_{\mathrm{v}} \frac{\partial \mathrm{p}^{\prime}}{\partial \mathrm{y}}\right)-\nabla \cdot\left(\rho^{*} \mathrm{v}^{*}\right)
$$

where the last term represents the mass imbalance, and the first two convection terms are unnecessary for incompressible flows. The pressure correction equation can be derived following the standard SIMPLE procedure [19]. In the present numerical method, all flow variables, except pressure, are located at the same grid points and the pressure node has been located at the centroid of the cell. The control volume for the pressure correction equation is defined as the cell enclosed by the four neighboring grid points. The velocity-pressure decoupling is eliminated by treating the pressure correction equation as a continuous form partial differential equation rather than treating it as a constraint condition. In the former case, the discrete pressure correction obtained from eq. (4) becomes a five-diagonal system of equations for rectangular grids. On the other hand, the discrete pressure correction equation obtalned by directly substituting the incremental pressure - incremental velocity relations into the conservation of mass equation yields a nine-diagonal system of equations. The latter discrete pressure correction equation can yield a velocity-pressure decoupled solution, whereas the former equation does not [17].

In control-volume based finite difference methods, the discrete system of equations is derived by integrating the governing differential equations over the control volume [19]. For curvilinear grids, the number of interpolations required to obtain flow variables at the cell boundaries is significantly reduced by using the present grid layout. Enhanced convergence rate is partly attributed to the grid layout which required 
fewer interpolations [17]. In solving the discrete system of equations, the off-diagonal terms are moved to the load vector term and the resulting system of equations can be solved using a tri-diagonal matrix algorithm (TDMA) .

\section{TURBULENCE EQUATIONS}

For clarity, the multiple-time-scale turbulence model supplemented with the near-wall turbulence model is summarized below. The turbulent kinetic energy and the energy transfer rate equations for the energy containing large eddies are given as;

$$
\begin{aligned}
& \left.u_{j} \frac{\partial k_{p}}{\partial x_{j}}-\frac{\partial}{\partial x_{j}}\left(\nu+\frac{\nu_{t}}{\sigma_{k p}}\right) \frac{\partial k_{p}}{\partial x_{j}}\right)-\operatorname{Pr}-\epsilon_{p} \\
& u_{j} \frac{\partial \epsilon_{p}}{\partial x_{j}}-\frac{\partial}{\partial x_{j}}\left(\left(\nu+\frac{\nu_{t}}{\sigma_{\epsilon}}\right) \frac{\partial \epsilon_{p}}{\partial x_{j}}\right)=c_{p 1} \frac{\operatorname{Pr}^{2}}{k_{p}}+c_{p} 2 \frac{\operatorname{Pr} \epsilon_{p}}{k_{p}}-c_{p} 3 \frac{\epsilon_{p}}{k_{p}}
\end{aligned}
$$

where the production rate is given as;

$$
P_{r}=\mu_{e}\left\{2\left(\frac{\partial u}{\partial x}\right)^{2}+2\left(\frac{\partial v}{\partial y}\right)^{2}+\left(\frac{\partial u}{\partial y}+\frac{\partial v}{\partial x}\right)^{2}\right\}
$$

The turbulent kinetic energy equation and the dissipation rate equations for the fine scale eddies are given as:

$$
\begin{aligned}
& u_{j} \frac{\partial k_{t}}{\partial x_{j}}-\frac{\partial}{\partial x_{j}}\left(\left(\nu+\frac{\nu_{t}}{\sigma_{k t}}\right) \frac{\partial k_{t}}{\partial x_{j}}\right)-\epsilon_{p}-\epsilon_{t} \\
& u_{j} \frac{\partial \epsilon_{t}}{\partial x_{j}}-\frac{\partial}{\partial x_{j}}\left(\left(\nu+\frac{\nu_{t}}{\sigma_{\epsilon t}}\right) \frac{\partial \epsilon_{t}}{\partial x_{j}}\right)=c_{t 1} \frac{\epsilon_{p} k_{t}}{k_{t}}+c_{t 2} \frac{\epsilon_{p} \epsilon_{t}}{k_{t}}-c_{t 3} \frac{\epsilon_{t}}{k_{t}}
\end{aligned}
$$


The turbulent kinetic energy equations, eqs. (5) and (7), are defined for the entire flow domain while the energy transfer rate and the dissipation rate equations are valid for the flow domain away from the near-wall region. The turbulence model constants are given as; $\sigma_{k p}-0.75, \sigma_{k t}=0.75$,

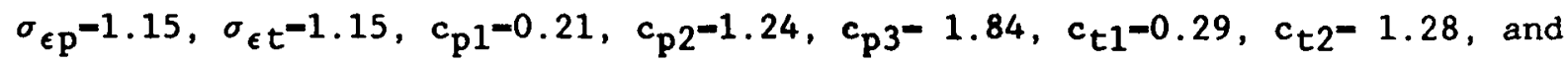
$c_{t 3}-1.66$. These turbulence model constants approximately satisfy the near-wall equilibrium turbulence condition, the decay rate of the grid turbulence [20], and the turbulence intensity growth rate in a constant shear flow [21]. Further discussion on the establishment of these turbulence model constants can be found in References 3 .

The energy transfer rate and the dissipation rate inside the near-wall layer are given as;

$$
\epsilon_{p}-\epsilon_{t}-\frac{\epsilon}{f_{\epsilon}}
$$

where

$$
\begin{aligned}
& \epsilon_{1}=\frac{c_{\mu \mathrm{f}^{3 / 4} \mathrm{k}^{3 / 2}}}{\kappa \mathrm{y}} \\
& \mathrm{f}_{\epsilon}=1-\exp \left(-\mathrm{A}_{\epsilon} \mathrm{R}_{\mathrm{t}}\right) \\
& \mathrm{R}_{t}=\frac{\mathrm{k}^{2}}{\nu \epsilon 1} \\
& \mathrm{~A}_{\epsilon}=\frac{\mathrm{c}_{\mu \mathrm{f}} \mathrm{f}^{3 / 2}}{2 \kappa^{2}}
\end{aligned}
$$

Note that $\epsilon_{1}$ in eq.(10) represents the standard dissipation rate for near-wall turbulent flows in equilibrium state. The dissipation rate given 
as eq. (9) is formally identical to the one proposed by Wolfshtein [12]. For $y \approx 0$, eq. (9) takes the limit value given as $2 \nu \mathrm{k} / \mathrm{y}^{2}$, which is an analytical solution of the turbulent kinetic energy equation for a limiting case as $y$ approaches the wall. Slightly away from the wall where the turbulence is in the equilibrium state, $f_{\epsilon}$ becomes unity. For near-wall equilibrium turbulent flows, the production rate $\left(P_{r}\right)$ is approximately equal to dissipation rate $\left(\epsilon_{t}\right)$ and hence the energy transfer rate $\left(\epsilon_{p}\right)$ from the low wave number production range to the high wave number dissipation range has to be approximately equal to the production and dissipation rates. Recall that the production rate vanishes on the wall and grows to a peak value at $y^{+} \approx 15$. Hence eq. (9) may not be a good approximation for $0<y^{+}<15$. However, use of the vanishing boundary condition for the turbulent kinetic energy on the wall yields a growth rate of turbulent kinetic energy and a production rate that are in good agreement with experimental data as well as theoretical analysis [9].

The eddy viscosity away from the near-wall layer is given as;

$$
\nu_{t}=c_{\mu f} \frac{k^{2}}{\epsilon_{p}}
$$

and that for the near-wall layer is given as;

$$
\nu_{t}=c_{\mu f} f_{\mu} \frac{k^{2}}{\epsilon_{1}}
$$

where $f_{\mu}=1-1 . / \exp \left(A_{1} \sqrt{ } R_{t}+A_{2} R_{t}{ }^{2}\right)$ is a linear function of the distance from the wall in the viscous sublayer and becomes unity in the fully turbulent region. $A_{1}=0.025$ and $A_{2}-0.00001$ have been used for the near wall 1ayer, see Reference 9. The eddy viscosity given as eq. (13) grows in proportion to 
the cubic power of the distance from the wall. It can be found in Reference 10 that the near-wall analysis yields the same growth rate of the eddy viscosity in the region very close to the wall. However, there also exist a few low Reynolds number turbulence models in which the eddy viscosity varies in proportion to the fourth power of the distance from the wall, see References 9 and 10 for more discussion.

\section{COMPUTATIONAL RESULTS}

The experimental data for the reattaching shear layer can be found in Reference 1. The inlet free stream velocity was $40 \mathrm{~m} / \mathrm{sec}$, the boundary layer thickness was 0.019 meters, and the helght of the backward-facing step was 0.0127 meters. The top wall was deflected from -2 degrees to 10 degrees to generate a strong adverse pressure gradient.

In numerical calculations, the inlet boundary was located at four step-heights upstream of the expansion corner and the exit boundary was located at approximately 35 step-heights downstream of the expansion corner. The flow domain was discretized by a 105 by 85 mesh with concentration of grid points near the expansion corner and in the bottom wall region, see Figure 2-(a). The grid in the vicinity of the expansion corner is fine enough to resolve details of the large eddies subjected to strong shear and sudden expansion; see Figure 2-(b). The inlet boundary conditions for the tangential velocity, the turbulent kinetic energies, and the dissipation rates ( $\epsilon_{p}$ and $\epsilon_{t}$ ) were obtained from experimental data for a fully developed boundary layer flow over a flat plate [3,22]. The non-dimensional velocity and the turbulent kinetic energy profiles were scaled to yield a boundary layer thickness of 0.019 meters at the inlet boundary. The no-slip boundary condition for velocities and vanishing 
turbulent kinetic energies were prescribed at the solid wall boundary. At the exit boundary, a vanishing gradient boundary condition was used for all flow variables except the pressure. A uniform pressure was prescribed at the exit boundary. The partition between the near-wall layer and the external region was located at approximately $\mathrm{y}^{+}=100,12$ grid points were allocated inside the near-wall layer. The mesh size of the first grid point on the bottom wall was $\Delta y^{+} \approx 2$ and the grid size in the normal to the wall direction was increased by a factor of approximately 1.15. Further details on the computational procedure can be found in Reference 17.

The calculated streamline contours are shown in Figure 3 . The flow field consisted of two recirculation zones. The primary recirculation zone extended from the separation corner toward the downstream direction; and the secondary recirculation zone was very small and confined in the corner region. The reattachment location versus the top wall deflection angle is shown in Figure 4. It can be seen in the figure that the $k-\epsilon$ and ARSM turbulence models largely under-predict the reattachment location. The modified ARSM yielded a significantly improved computational result, however, the present computational result compared more favorably with the measured data than did the modified ARSM.

The static pressure contour lines are shown in Figure 5, where the pressure has been normalized by the inlet total pressure and the incremental pressure between the contour lines is 0.005 . It can be seen in the figure that a few contour lines pass through the expansion corner, and thus there exists a mild base pressure in the backward-facing step region. The calculated static pressure on the wall is compared with experimental data as well as the numerical results of Reference 1 in Figure 6 . The mild pressure drop at $x / H=0$ represent the base pressure. For $\alpha=0^{0}$, the present 
computational result compared slightly more favorably with the measured wall pressure than the other computational results. For $\alpha-6^{\circ}$, all the computational results compared decently with the measured data; however, the slope of the wall pressure in the continuously diverging downstream region obtained in the present study compared more favorably with the measured data than the other results. This difference may due to the different numerical methods used.

The calculated wall shearing stresses are shown in Figure 7 . It can be seen in the figure that the location of the peak wall shearing stress obtained using the $\mathrm{k}-\epsilon$ turbulence model is grossly in error. It is interesting to note that the modified ARSM under-predicts the peak value and the present turbulence model over-predicts the peak value even though the relative differences are almost the same for both deflection angles.

The mean velocity, the turbulent kinetic energy, and the Reynolds stress profiles at four downstream locations are compared with experimental data and with the calculated results using the modified ARSM [1,2] in Figures 8-10, respectively. The experimental turbulent kinetic energy shown in Figure 9 was estimated using the measured value of $u^{\prime 2}+v^{\prime 2}$ and an assumption that $w^{\prime 2}=\left(u^{\prime 2}+v^{\prime 2}\right) / 2$. As shown in Figures $8-10$, both computational results exhibit fair comparison with the experimental data. It can be seen in Figure 9 that the peak value of the turbulent kinetic energy and the shape of the turbulent kinetic energy profile obtained using the multiple-time-scale turbulence model compare slightly better with the measured data than those obtained using the modified ARSM at $\mathrm{x} / \mathrm{H}=1.0$ where the turbulence is in a strongly inequilibrium state. It has been shown previously that the improved computational results for complex turbulent flows are attributed to the capability of the multiple-time-scale 
turbulence model to resolve the inequilibrium turbulence [3]. The same argument can be applied for the present flow case. At further downstream locations, the present computational results compared slightly less favorably with the experimental data, a result perhaps due to the near-wall turbulence model which can not take into account of the inequilibrium turbulence.

The ratio of turbulent viscosity to molecular viscosity at three downstream locations are shown in Figure 11. It can be seen in the figure that the Jones-Launder $k-\epsilon$ turbulence model over-estimates the ratio so that the reattachment location is largely under-predicted. On the other hand, the present computational results compare quite favorably with the measured data so that the reattachment location is correctly predicted. The calculated production and dissipation rates of the turbulent kinetic energy at the same downstream locations were qualitatively and quantitatively almost the same as those of reference 1 .

\section{CONCLUSIONS}

Numerical calculations of reattaching shear layers in a diverging channel using a multiple-time-scale turbulence model supplemented with a near-wall turbulence model have been presented. The calculated reattachment location versus the top wall deflection angle obtained using the present turbulence model was in excellent agreement with measured data. The calculated wall pressure and the wall shearing stress were also in good agreement with the experimental data. The rest of the present computational results such as the normalized velocity profiles and the Reynolds stress profiles compared favorably with experimental data. The computational results obtained using the multiple-time-scale turbulence model compared 
slightly more favorably with the experimental data than those obtained using the modified algebraic Reynolds stress turbulence model. It has been shown that prediction of the correct reattachment location depends on the prediction of the correct level of the turbulent viscosity, which depends, in turn, on the capability of a turbulence model to resolve the entire turbulence structure of the flow field correctly. Thus the improved computational results are attributed to the capability of the multiple-time-scale turbulence model to resolve the strong inequilibrium turbulence in the vicinity of the expansion corner and in the following shear-layer region. 


\section{REFERENCES}

1. D. M. Driver and H. L. Seegmiller, "Features of a Reattaching Turbulent Shear Layer in Divergent Channel Flow, " J.AIAA, vol. 23, pp. 163-171, 1985.

2. M. Sindir, "Numerical Study of Separating and Reattaching Flows in a Backward-Facing Step Geometry," Ph.D. Thesis, University of California at Davis, CA, 1982.

3. S.-W. Kim and C.-P. Chen, "A Multiple-Time-Scale Turbulence model Based on Variable Partitioning of the Turbulent Kinetic Energy Spectrum", To appear in the Numerical Heat Transfer, 1989, Also available as NASA CR-179222, 1987 and AIAA Paper 88-0221, 1988.

4. S.-W. Kim and C.-P. Chen, "A Review on Various Flow-Solid Interaction Analysis Methods with Emphasis on Recent Advances in Turbulence Models and Flow Analysis Methods," AIAA Paper 88-3685, 1988.

5. K. Hanjelic, B. E. Launder, and R. Schiestel, "Multiple-Time-Scale Concepts in Turbulent Shear Flows" in L. J. S. Bradbury, F. Durst, B. E. Launder, F. W. Schmidt, and J. H. Whitelaw, (eds.), Turbulent Shear Flows, vol. 2, pp. 36-49, Springer-Verlag, New York, 1980.

6. R. Schiestel, "Multiple-Time-Scale Modeling of Turbulent Flows in One point Closure", Physics of Fluids, vol. 30, pp. 722-731, 1987.

7. R. Schieste1, "Multiple-Scale Concept in Turbulence Modelling, II. Reynolds Stresses and Turbulent Fluxes of a Passive Scalar, Algebraic Modelling and Simplified Model using Boussinesq Hypothesis", Journal de Mechanique theorique et appliquee, vol. 2, pp. 601-628, 1983.

8. S. W. Tu and B. R. Ramaprian, "Fully Developed Periodic Turbulent Pipe Flow, Part I. Main Experimental Results and Comparison with 
Predictions," J.Fluid Mech., vol. 137, pp. 31-58, 1983.

9. S.-W. Kim, "A Near-Wall Turbulence Model and Its Application to Fully Developed Turbulent Channel and Pipe Flows," To appear in Numerical Heat Transfer, 1989. Also avallable as NASA TM-101399, 1988.

10. V. C. Patel, W. Rodi and G. Scheuerer, "Turbulence Models for Near Wall and Low Reynolds Number Flows: A Review", J. AIAA, 23, pp. $1308-1319(1985)$.

11. R. S. Amano, "Development of a Turbulent Near-Wall Model and Its Application to Separated and Reattached Flows," Numerical Heat Transfer, vol. 7, pp. 59-75, 1984.

12. M. Wolfshtein, "The Velocity and Temperature Distribution in One-Dimensional Flow with Turbulence Augmentation and Pressure Gradient," Int. J. Heat and Mass Iransfer, vol. 12, pp. 301-318, 1969.

13. H. C. Chen and V. C. Patel, "Practical Near-Wall Turbulence Models for Complex Flows Including Separation," AIAA-87-1300, 1987.

14. A. Roshko, "Structure of Turbulent Shear Flows: A New Look," J, AIAA, vol. 14, No. 10, pp. $1349-1357,1976$.

15. Gibson, M. M., Spalding, D. B., and Zinser, W., "Boundary Layer Calculations using the Hassid-Poreh One-Equation Energy Model," Letters in Heat and Mass Transfer, vol. 5, pp. 73-80, 1978.

16. Hassid, S., and Poreh, M., "A Turbulent Energy Model for Flows with Drag Reduction," J.Fluid Engineering, Transactions of ASME, pp. $234-241,1975$.

17. S.-W. Kim, "A Control-Volume Based Reynolds Averaged Navier-Stokes Equation Solver Valid at All Flow Velocities," NASA TM-101488, 1989.

18. S. -W. Kim, "Numerical Computation of Shock Wave - Turbulent Boundary Layer Interaction in Transonic Flow over an Axisymmetric Curved Hill," 
NASA TM-101473, 1989.

19. S. V. Patankar, Numerical Heat Transfer and Fluid Flow, McGraw-Hill, New York, 1980.

20. F. H. Harlow and P. I. Nakayama, "Transport of Turbulence Energy Decay Rate," Los Alamos Sci. Lab., LA-3854, 1968.

21. V. G. Harris, J. A. H. Graham, and S. Corrsin, "Further

Experiments in Nearly Homogeneous Turbulent Shear Flow," J,Fluid Mech., vol. 81, pp. 657-687, 1977.

22. P. S. Klebanoff, "Characteristics of Turbulence in a Boundary Layer with Zero Pressure Gradient," NACA Report 1247, 1955. 


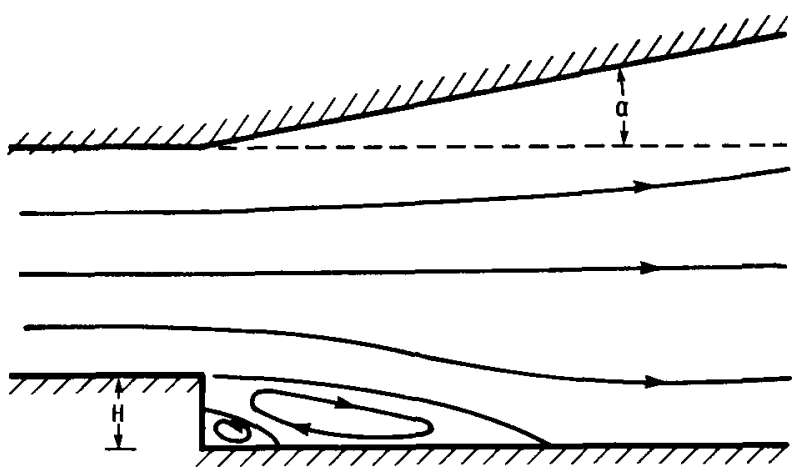

FIGURE 1. - MOMENCLATURE OF THE REATTACHING SHEAR LAYER,

H: HEIGHT OF THE BACKHARD-FACING STEP, a: TOP WALL DEFLECTION ANGLE.

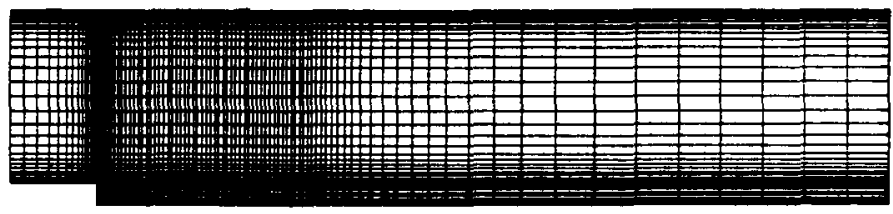

(a) ENTIRE DOMAIN.

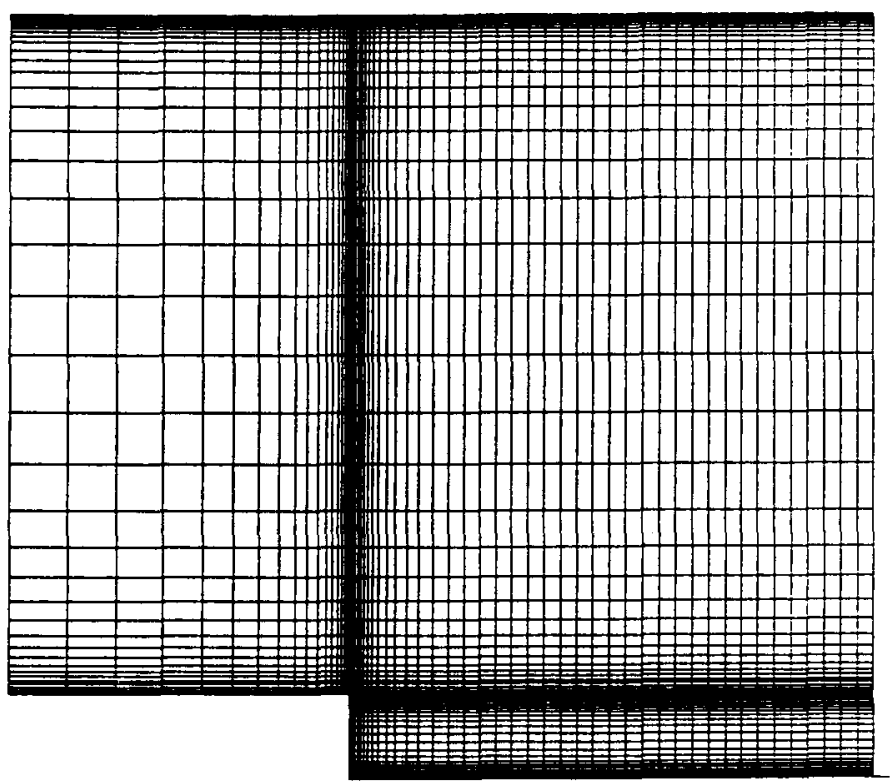

(b) VICINITY OF EXPANSION CORMER.

FIGURE 2. - DISCRETIZATION OF FLON DOMIM.

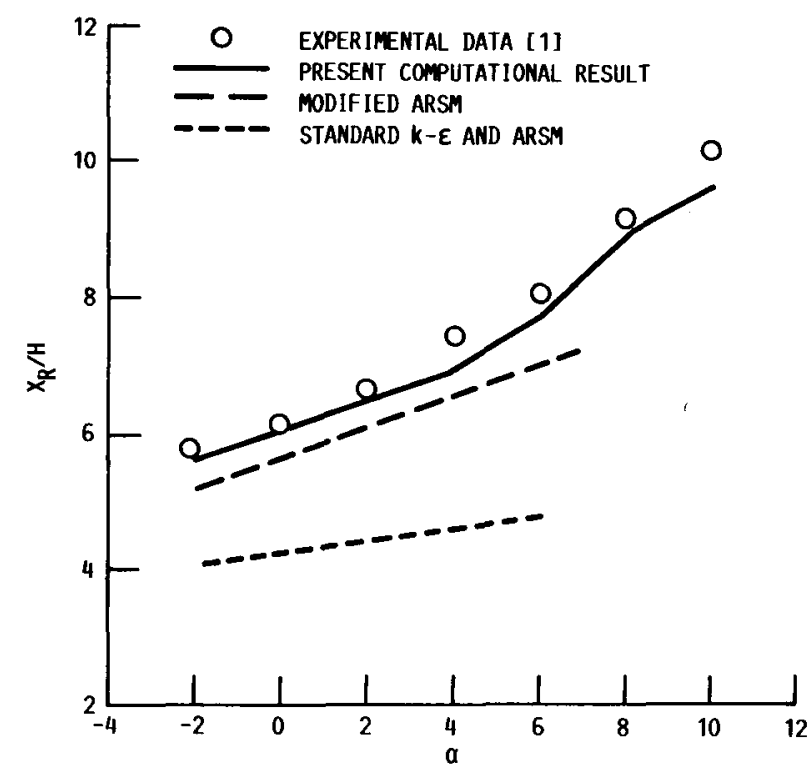

FIGURE 4. - REATTACHIENT LOCATION VERSUS DEFLECTION ANGLE. 


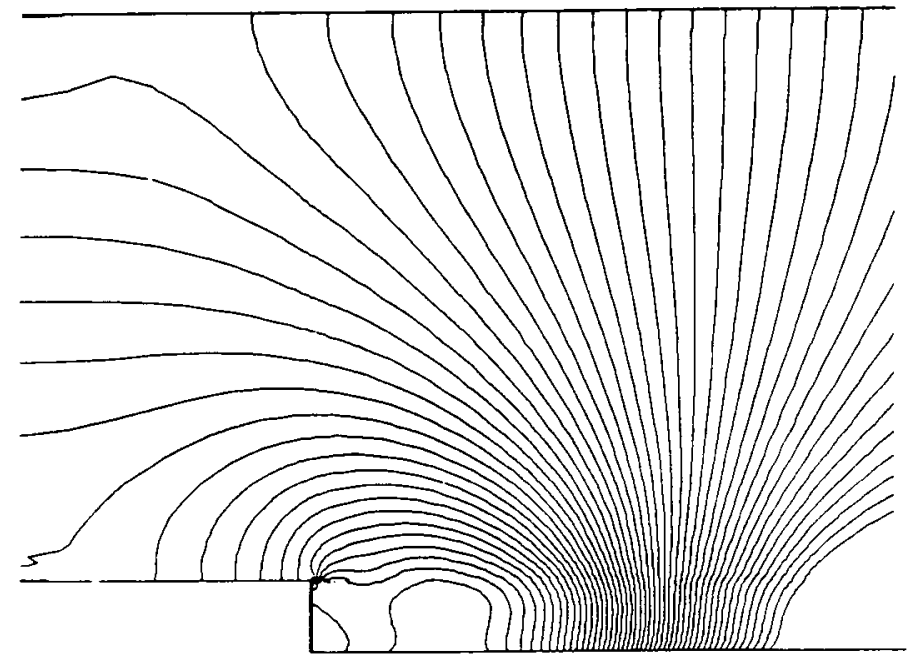

FIGURE 5. - PRESSURE CONTOUR.
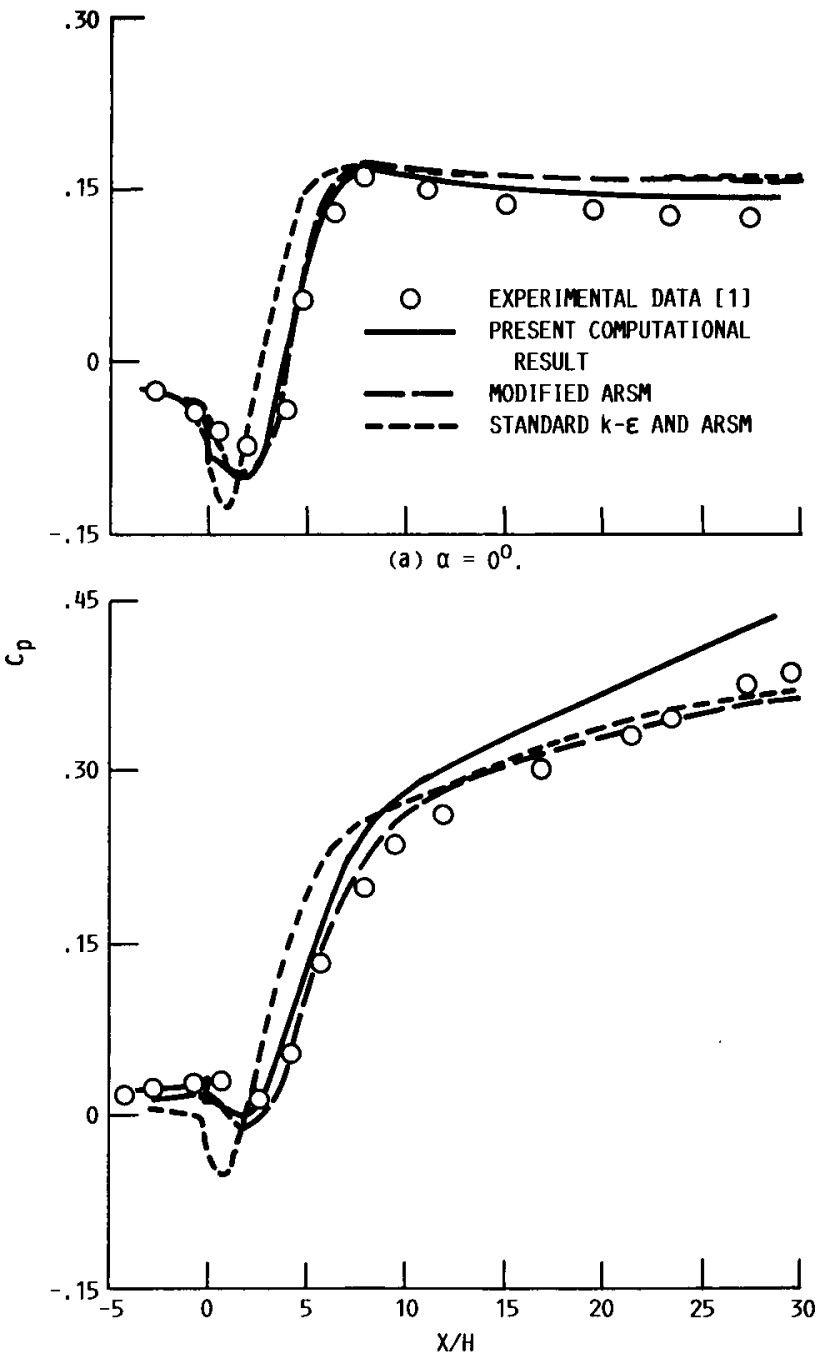

(b) $a=6^{\circ}$.

FIGURE 6. - PRESSURE ON BOTTON MALL. 


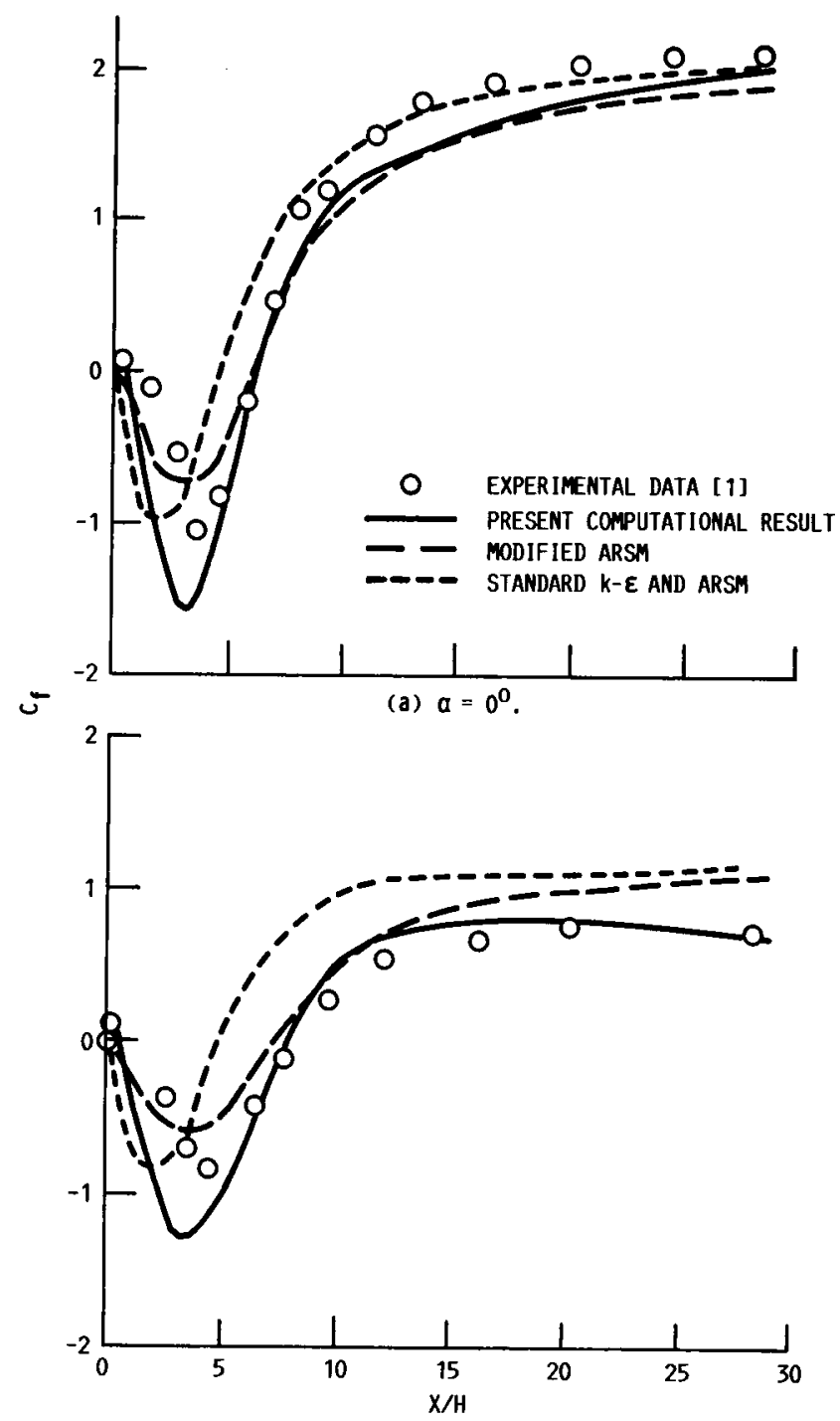

(b) $a=6^{0}$.

FIGURE 7. - SHEAR STRESS ON BOTTOM WALL.

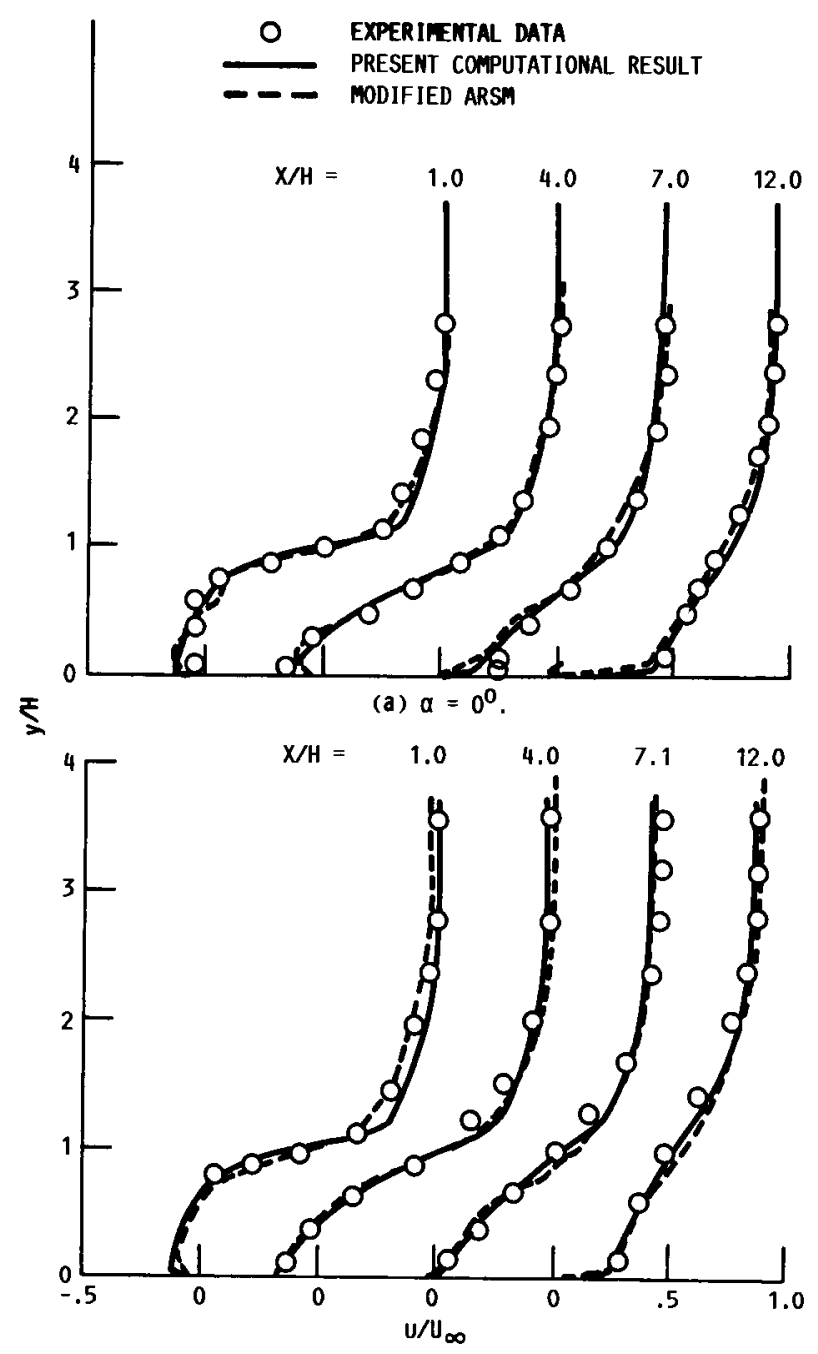

(b) $a=6^{\circ}$.

FIGURE 8. - VELOCITY PROFILES. 


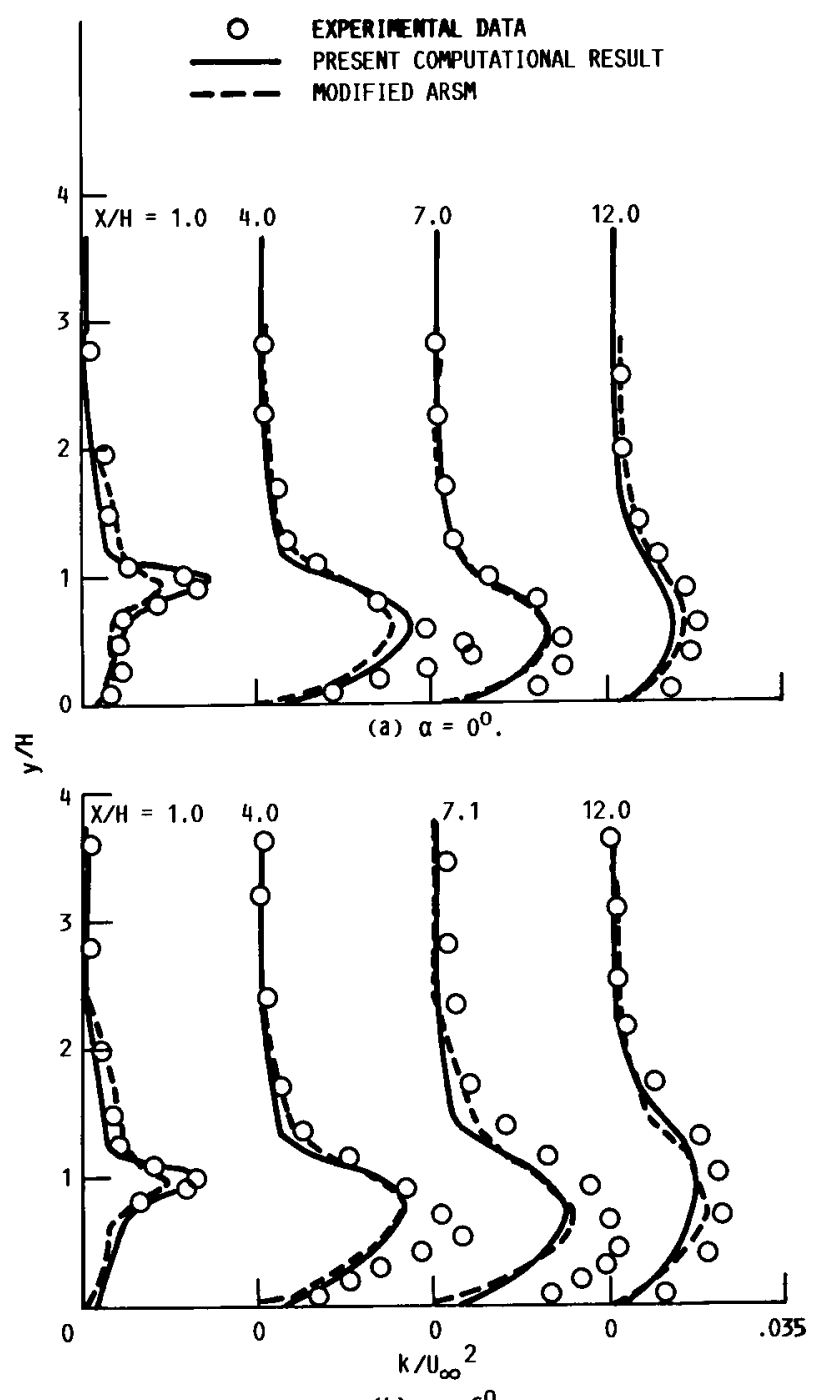

(b) $a=6^{0}$.

FIGURE 9. - TURBULENT KINETIC ENERGY PROFILES.
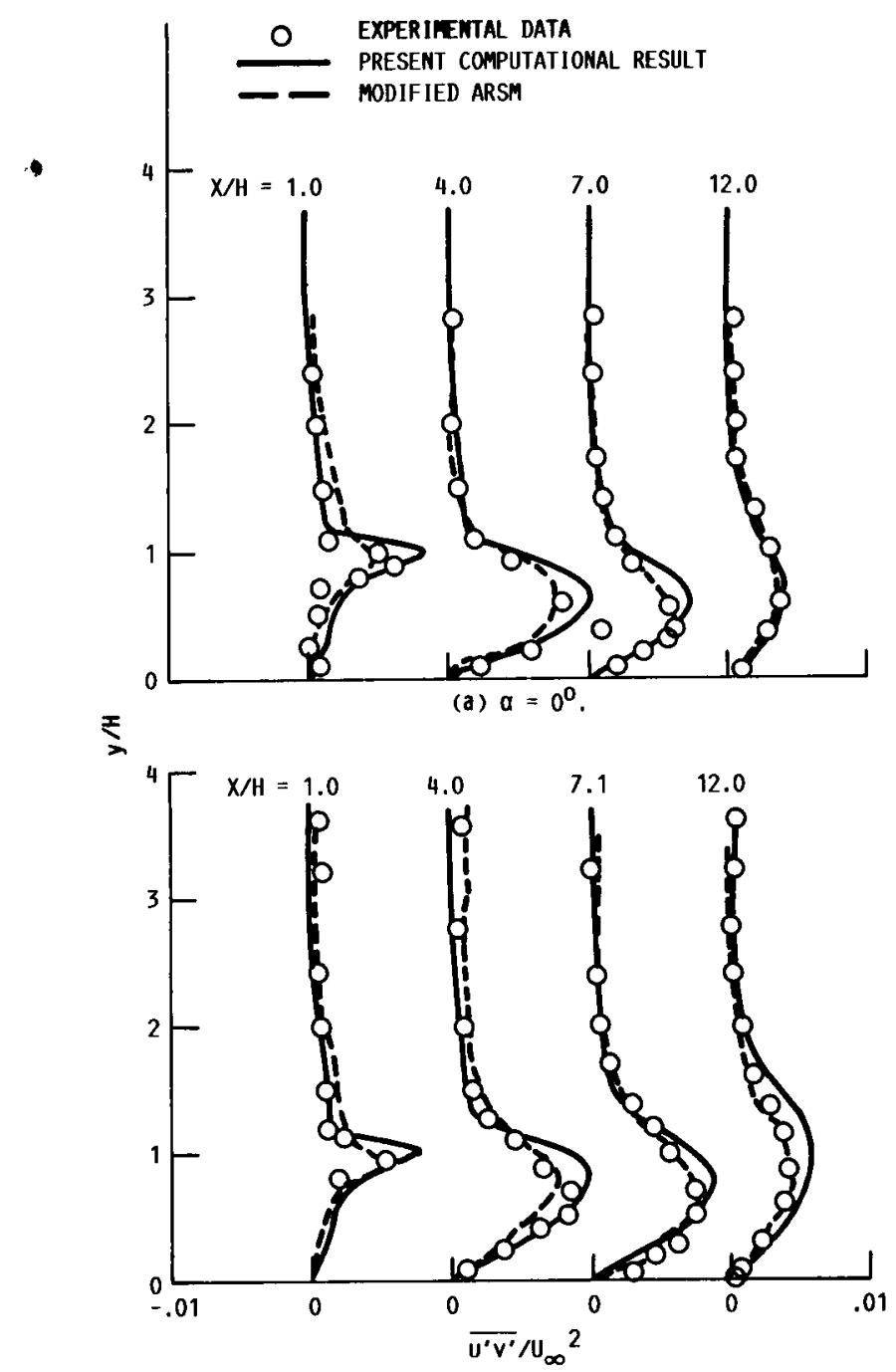

(b) $a=6^{0}$.

FIGURE 10. - REYMOLDS STRESS PROFILES. 


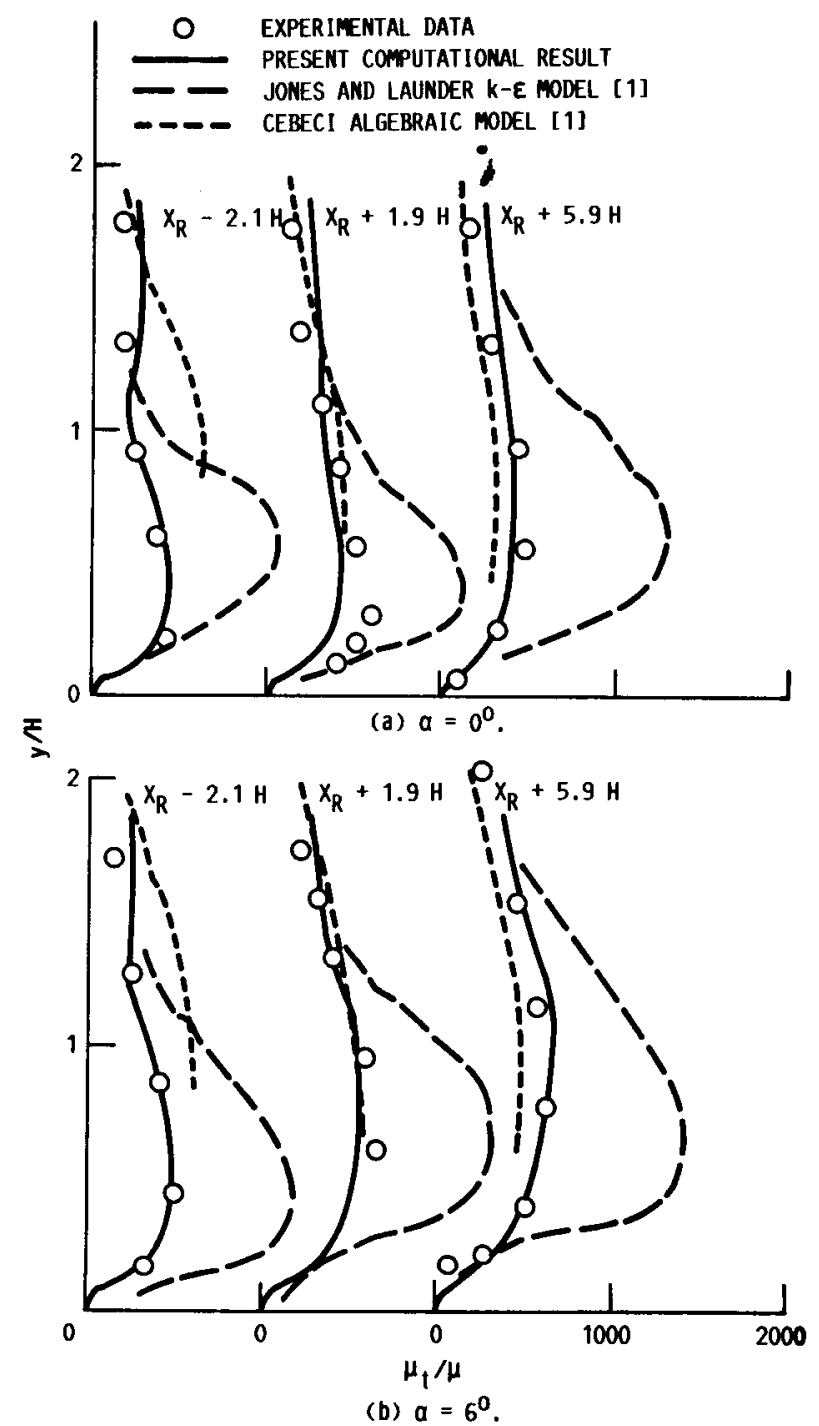

FIGURE 11. - RATIOS OF TURBULENT VISCOSITY OVER MOLECULAR VISCOSITY. 


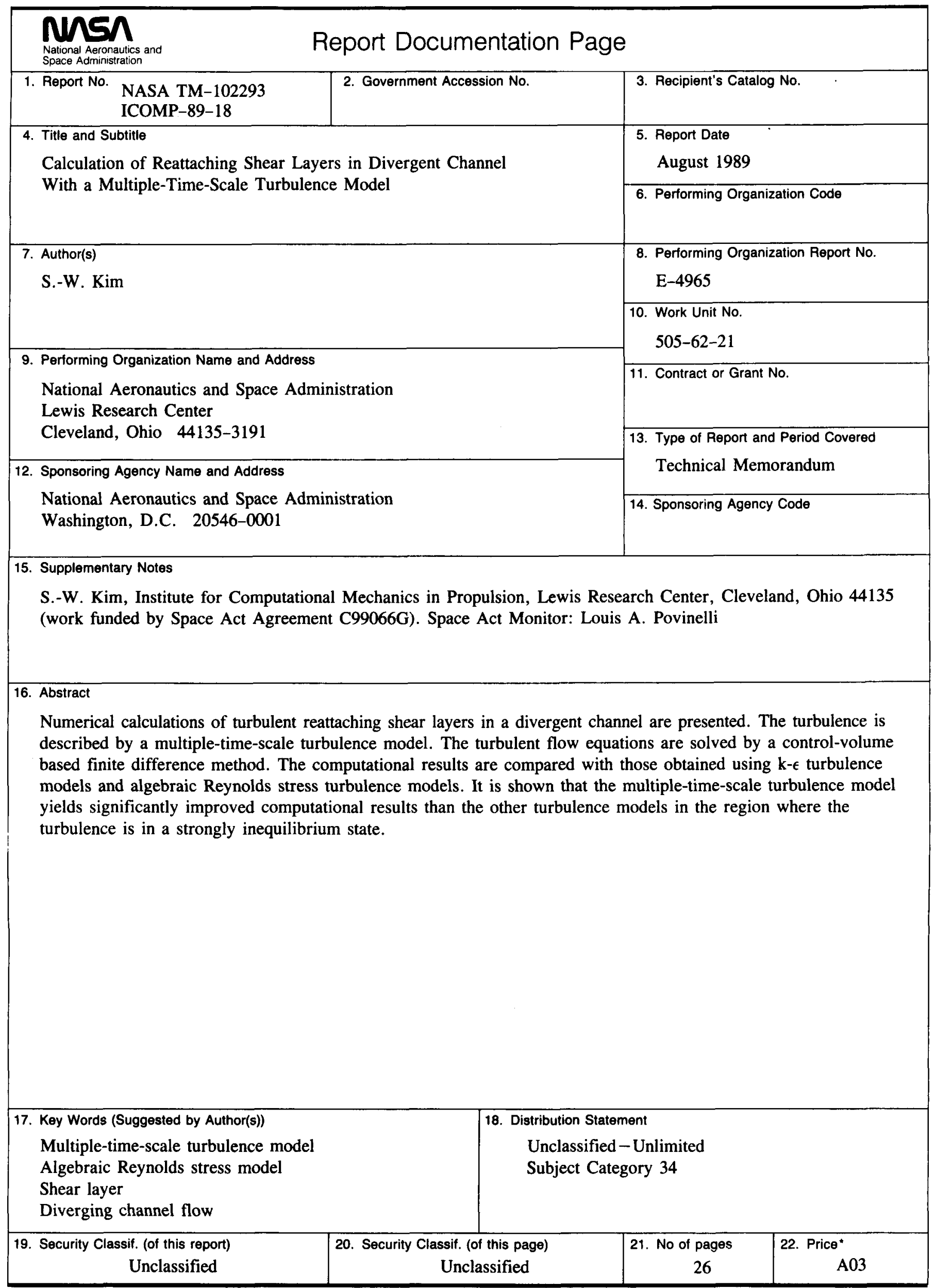

